\title{
Thermodynamic geometry of the quark-meson model
}

\author{
Bonan Zhang, Shen-Song Wan $\odot$, and Marco Ruggieri* \\ School of Nuclear Science and Technology, Lanzhou University, \\ 222 South Tianshui Road, Lanzhou 730000, China
}

(Received 31 July 2019; published 22 January 2020)

\begin{abstract}
We study the thermodynamic geometry of the quark-meson model, focusing on the curvature, $R$, around the chiral crossover at finite temperature and baryon chemical potential. We find a peculiar behavior of $R$ in the crossover region, in which the sign changes and a local maximum develops; in particular, the height of the peak of $R$ in the crossover region becomes large in the proximity of the critical end point and diverges at the critical end point. The appearance of a pronounced peak of $R$ close to the critical end point supports the idea that $R$ grows with the correlation volume around the phase transition. Moreover, the change of sign of $R$ in the crossover region supports the idea that an attractive interaction develops at the mesoscopic level in that range of temperature. We also analyze the mixed fluctuations of energy and baryon number, $\langle\Delta U \Delta N\rangle$, which grow up substantially in the proximity of the critical end point: In the language of thermodynamic geometry these fluctuations are responsible for the vanishing of the determinant of the metric, which results in thermodynamic instability and are thus related to the appearance of a critical end point.
\end{abstract}

DOI: 10.1103/PhysRevD.101.016014

\section{INTRODUCTION}

An interesting idea of statistical mechanics is that of a metric in the manifold spanned by the thermodynamic variables: This is related to the theory of fluctuations among equilibrium states and leads to the concept of thermodynamic geometry and thermodynamic curvature [1-44]. For example, in the grand-canonical ensemble the equilibrium state is specified as long as the intensive independent variables like temperature, chemical potential and others are fixed, and physical quantities like energy and particle number fluctuate with the probability given by the Gibbs ensemble. Considering the pair of intensive variables $\left(\beta^{1}, \beta^{2}\right)$ the probability of a fluctuation from $S_{1}=\left(\beta^{1}, \beta^{2}\right)$ to $S_{2}=\left(\beta^{1}+d \beta^{1}, \beta^{2}+d \beta^{2}\right)$ is proportional to

$$
\sqrt{g} \exp \left(-\frac{1}{2} g_{i j} d \beta^{i} d \beta^{j}\right)
$$

where $g_{i j} \equiv \partial^{2} \log \mathcal{Z} / \partial \beta^{i} \partial \beta^{j}$ is called the thermodynamic metric tensor, $g=\operatorname{det}\left(g_{i j}\right)$ is the determinant of $g_{i j}$ and $\mathcal{Z}$ is the grand-canonical partition function. It is therefore natural to define the line element $d \ell^{2}=g_{i j} d \beta^{i} d \beta^{j}$ which measures effectively a distance between $S_{1}$ and $S_{2}$, in the

\footnotetext{
"ruggieri@1zu.edu.cn
}

Published by the American Physical Society under the terms of the Creative Commons Attribution 4.0 International license. Further distribution of this work must maintain attribution to the author(s) and the published article's title, journal citation, and DOI. Funded by SCOAP ${ }^{3}$. sense that a large $d \ell^{2}$ corresponds to a small probability of a fluctuation from $S_{1}$ to $S_{2}$. With the aid of $g_{i j}$ it is possible to define the thermodynamic curvature, $R=2 R_{1212} / g$ with $g=\operatorname{det}\left(g_{i j}\right)$ and $R_{1212}$ corresponding to the only independent component of the Riemann tensor for a twodimensional manifold. As it is clear from the very definition of $g_{i j}$, the thermodynamic curvature depends on the second and third order moments of the thermodynamic variables that are conjugated to $\left(\beta^{1}, \beta^{2}\right)$; therefore it carries information about the fluctuation of the physical quantities in particular around a phase transition, where these fluctuations are expected to be very large. For example, if $\left(\beta^{1}, \beta^{2}\right)=(1 / T,-\mu / T)$ then $R$ contains information about the fluctuations of energy and particle number. One of the merits of the thermodynamic curvature is that in the proximity of a second order phase transition $|R| \propto \xi^{d}$ where $d$ denotes the spatial dimension and $\xi$ is the correlation length: As a consequence, it is possible to grasp information about the correlation length by means of thermodynamics only. This divergence is related to the vanishing of the determinant of the metric; therefore the thermodynamic geometry gives information on the location of the phase transition in the $\left(\beta^{i}\right)$ space.

The main purpose of this article is to report on our study of the thermodynamic geometry, and in particular of the thermodynamic curvature, of the quark-meson (QM) model of quantum chromodynamics (QCD); see [45-48] and references therein. This model is used to describe chiral symmetry breaking in vacuum, as well as chiral symmetry restoration at finite temperature $T$ and baryon chemical potential $\mu$; it can be used to model, for example, the QCD 
phase transition after the big bang, as well as the cold and dense quark matter presumably present in the core of compact stellar objects. In these applications usually a positive value of $\mu$ is considered since matter with finite baryon density is modeled, while a negative $\mu$ would give a finite antibaryon density.

Although the phase diagram of this model is well known within the community of quark-gluon plasma physicists, we summarize it in order to give a qualitative understanding of the phases and transition lines at finite temperature and chemical potential. In the QM model, we introduce both quarks and mesons in the Lagrangian; the meson potential is such that the $O(4)$ symmetry is broken spontaneously down to $O(3)$ at $T=\mu=0$, leaving one massive $\sigma$ meson and an isotriplet of pions, as well as a mean field value for the $\sigma$ field. The latter couples to quarks via the interaction $\sigma \bar{\psi} \psi$, which results in a nonvanishing chiral condensate $\langle\bar{\psi} \psi\rangle \neq 0$. At finite $T$ and $\mu$ chiral symmetry is restored due to thermal fluctuations that destroy the mean field, and at zero chemical potential this transition is a second order one, with an order parameter that vanishes exactly above the critical temperature and the transition happens continuously. In the chiral limit, the symmetry breaking pattern/ restoration is exact. However, an explicit but soft chiral symmetry breaking term is introduced in the Lagrangian to gives mass to pions: As a consequence, chiral symmetry is broken explicitly, the mean field never vanishes although becoming very small at high temperature, and the transition becomes a smooth crossover. This situation is very similar to that encountered in statistical models of ferromagnetism, in which a magnetic field always induces a finite value for the average spin although the temperature is higher than the Curie temperature: In the case of the QM model, as well as in QCD, the mass term acts like the magnetic field and the spin polarization is played by the condensate of the $\sigma$ field. At high chemical potential the change of the condensate with the temperature is no longer smooth: This is interpreted as the presence of a critical end point in the phase diagram, at which a first order and the crossover lines meet. This point is identified with a second order phase transition since at this point, and only at this point, the susceptibilities diverge as they would at a second order phase transition; see [45-48] and references therein, as well as the results shown in the present article.

These considerations are summarized in Fig. 1 in which we show the phase diagram of the QM model at finite $T$ and $\mu$, where $\mu$ corresponds to the baryon chemical potential. The labels $\chi_{S B}$ and $\chi_{S R}$ denote the phases in which chiral symmetry is broken spontaneously and (approximately) restored respectively. The first order and crossover lines meet at the critical end point labeled $C E P$.

The phase diagram of the QM model fits the expectations of QCD well. In fact, it is well known that at zero baryon chemical potential, QCD matter experiences a smooth crossover from a low temperature confined phase in which

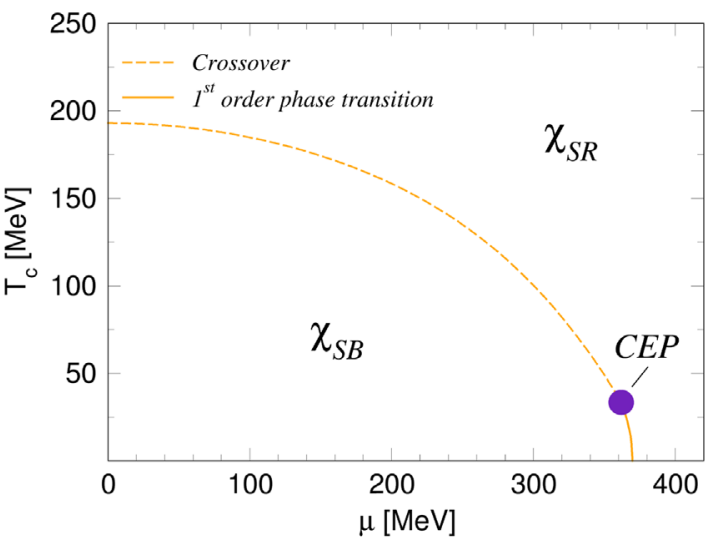

FIG. 1. Phase diagram of the quark-meson model at finite $T$ and $\mu$. The labels $\chi_{S B}$ and $\chi_{S R}$ denote the phases in which chiral symmetry is broken spontaneously and (approximately) restored respectively. The first order and crossover lines meet at the critical end point labeled $C E P$.

chiral symmetry is spontaneously broken, to a high temperature phase in which color is deconfined and chiral symmetry is approximately restored [49-53]. The situation is however unclear at finite baryon chemical potential for QCD with three colors, due to the sign problem that forbids first principle calculations. Because of this, effective models like the QM model have been used to study the phase structure of QCD at finite $\mu$ : Nowadays there is consensus that the smooth crossover becomes a first order phase transition if $\mu$ is large enough, suggesting the presence of the critical end point in the $(T, \mu)$ plane at which the crossover becomes a true phase transition with divergent susceptibilities, and this point marks the separation between the crossover on the one hand and the first order line on the other hand; see for example [54,55] for reviews.

We consider here the QM model at finite $T$ and $\mu$, which has been applied many times to study the phase structure of QCD, and we study its thermodynamic geometry following the lines depicted in [43] where a similar study has been performed for the Nambu-Jona-Lasinio (NJL) model. The advantage of using the QM model is its renormalizability, which removes the dependence of the results on the effective ultraviolet cutoff that instead appears in NJL calculations. Moreover, it is interesting to check how the predictions of the phase structure of QCD change when different effective models are used: This can not only shed light on the qualitative picture, but also put a quantitative statement on the theoretical uncertainty of model predictions, for example on the location of the CEP.

We can anticipate the main results here. The curvature is found to be negative in almost all ranges of $T$ and $\mu$ explored here, both at low temperature where the mean field is nonzero and quarks are quite massive, and at very high temperature where quarks are almost massless, in agreement with an ideal fermion gas. A change of sign is 
observed near the chiral crossover, where $R$ develops a local maximum which becomes more pronounced when the chemical potential is increased. Moreover, the dependence of $R$ on the temperature is nontrivial for large $\mu$ where two peaks are found in the crossover region, one negative at smaller temperature and one positive at higher temperature. A change of sign of $R$ has been observed for many substances [18,20,22,23,25,27-29,31] and it has been interpreted in terms of the nature of the attractive/repulsive microscopic interaction. This suggests that at the crossover the interaction at a mesoscopic level changes, leading to an effective attractive interaction; below and above the crossover, the fermion nature of the system dominates instead and the curvature is negative. Moreover, the height of the peak of $R$ increases along the critical line as $\mu$ is increased from zero to the corresponding CEP value and diverges at the CEP: This is in agreement with $|R| \propto \xi^{3}$ since the correlation length remains finite at the crossover but increases as the crossover becomes sharper and eventually diverges at the critical end point. We also discuss how the mixed susceptibility, $\langle\Delta U \Delta N\rangle$ which is nonzero at finite $\mu$, is crucial to have $g=0$ at the CEP.

The plan of the article is as follows. In Sec. II we briefly review the thermodynamic geometry and in particular the thermodynamic curvature. In Sec. III we review the QM model. In Sec. IV we discuss $R$ for the QM model. Finally, in Sec. V we draw our conclusions. We use the natural units system $\hbar=c=k_{B}=1$ throughout this article.

\section{THERMODYNAMIC CURVATURE}

The idea of thermodynamic fluctuations, thermodynamic geometry and in particular of thermodynamic curvature is pretty old [1,2] and is nowadays it is introduced in several textbooks of statistical mechanics; see for example $[14,56,57]$. We present here only the few concepts that are closely related to our work, while we refer to $[15,19]$ and references therein for more details.

Let us consider a thermodynamics system in the grandcanonical ensemble: We assume that its thermodynamic state at equilibrium is specified in terms of the coordinates $(T, \mu)$, where $T$ is the temperature and $\mu$ is the chemical potential conjugated to the particle density. Alternatively we can use a different set of coordinates, namely $(\beta, \gamma)$ where $\beta=1 / T$ and $\gamma=-\mu / T$. It is possible to build up a metric space in the $(\beta, \gamma)$ manifold by defining a distance, namely

$$
d \ell^{2}=g_{\beta \beta} d \beta d \beta+2 g_{\beta \gamma} d \beta d \gamma+g_{\gamma \gamma} d \gamma d \gamma,
$$

where for classical systems with grand-canonical partition function $\mathcal{Z}$ [38]

$$
g_{i j} \equiv \frac{\partial^{2} \log \mathcal{Z}}{\partial \beta^{i} \partial \beta^{j}}=\frac{\partial^{2} \phi}{\partial \beta^{i} \partial \beta^{j}} \equiv \phi_{, i j},
$$

and $\phi \equiv \beta P, P=-\Omega$ with $\Omega$ representing the thermodynamic potential per unit volume; moreover, $\beta^{1}=1 / T$, $\beta^{2}=\gamma=-\mu / T$. The comma denotes a partial derivative as usual. The line element $d \ell^{2}$ in Eq. (2) represents effectively a distance in the two-dimensional manifold, in the sense that the probability to fluctuate from the equilibrium state $S_{1}=(\beta, \gamma)$ to another equilibrium state $S_{2}=(\beta+d \beta, \gamma+d \gamma)$ is

$$
P \propto \exp \left(-\frac{d \ell^{2}}{2}\right)
$$

therefore, the larger the distance the less probable it is to have a fluctuation from $S_{1}$ to $S_{2}$ and the two states are effectively distant. In the above equation $g$ denotes the determinant of the metric tensor in Eq. (3). With these definitions the thermodynamic curvature of the two-dimensional manifold is given by

$$
R=-\frac{1}{2 g^{2}}\left|\begin{array}{ccc}
\phi_{, \beta \beta} & \phi_{, \beta \gamma} & \phi_{, \gamma \gamma} \\
\phi_{, \beta \beta \beta} & \phi_{, \beta \beta \gamma} & \phi_{, \beta \gamma \gamma} \\
\phi_{, \beta \beta \gamma} & \phi_{, \beta \gamma \gamma} & \phi_{, \gamma \gamma \gamma}
\end{array}\right| \text {, }
$$

where $\|$ means the determinant of the $3 \times 3$ matrix. We notice that our sign convention agrees with [38], in particular $R>0$ for the sphere. For our choice of coordinates we have, for example [36],

$$
\begin{gathered}
\phi_{, \beta \beta}=\left\langle(U-\langle U\rangle)^{2}\right\rangle, \\
\phi_{, \beta \gamma}=\langle(U-\langle U\rangle)(N-\langle N\rangle)\rangle, \\
\phi_{, \gamma \gamma}=\left\langle(N-\langle N\rangle)^{2}\right\rangle,
\end{gathered}
$$

where $U, N$ denote the internal energy and the particle number respectively. The diagonal matrix elements $\phi_{, \beta \beta}$ and $\phi_{, \gamma \gamma}$ are related to the specific heat and the isothermal compressibility, $\chi_{T}$, respectively [36], namely

$$
\begin{aligned}
\beta^{2} \phi_{, \beta \beta} & =\left(\frac{\partial U}{\partial T}\right)_{\gamma}, \\
\beta \phi_{, \gamma \gamma} & =\frac{N^{2}}{V} \chi_{T},
\end{aligned}
$$

where $\chi_{T}=-(\partial V / \partial P)_{T}$.

The thermodynamic curvature has the merit that close to a phase transition $|R| \propto \xi^{3}$ in three spatial dimensions, where $\xi$ corresponds to the correlation length. Therefore, in principle it is possible to access microscopic details like $\xi$ in the proximity of the phase transition just by means of thermodynamics. Within our sign convention, $R<0$ for an ideal fermion gas and $R>0$ for an ideal boson gas; $R=0$ for the ideal classical gas; for anyon gases $[32,33]$ it is 
possible to deform continuously the distribution from a fermionic to a bosonic one, and this results in a change of the sign of $R$ in agreement with the previous statement. For interacting systems the interpretation of the sign of $R$ is far more complicated and nowadays there is no consensus on what this sign means. For example, for many substances it has been found that $R<0$, but for these there exists a range of temperature/density in which $R>0$ and this change of sign has been interpreted as a transition from a fluid to a solidlike fluid behavior [20,28]. In addition to this, it has been found that $R>0$ if the attractive interaction dominates, while $R<0$ if the repulsive interaction is more important [23]: While this seems to be satisfied by several substances, it is unclear if this relation between the sign of $R$ and the nature of the interaction is general.

\section{THE QUARK-MESON MODEL}

The QM model is an effective model of QCD in which quarks and mesons are considered on the same footing; it is a very well-known model in quantum field theory, where it is called the linear-sigma model coupled to fermions (see e.g., $[58,59])$. The meson part of the Lagrangian density of the QM model is

$\mathcal{L}_{\text {mesons }}=\frac{1}{2}\left(\partial^{\mu} \sigma \partial_{\mu} \sigma+\partial^{\mu} \pi \cdot \partial_{\mu} \pi\right)-\frac{\lambda}{4}\left(\sigma^{2}+\pi^{2}-v^{2}\right)^{2}+h \sigma$,

where $\pi=\left(\pi_{1}, \pi_{2}, \pi_{3}\right)$ corresponds to the pion isotriplet field. This Lagrangian density is invariant under $O(4)$ rotations. On the other hand, as long as $v^{2}>0$ the potential develops an infinite set of degenerate minima. We choose one ground state, namely

$$
\langle\boldsymbol{\pi}\rangle=0, \quad\langle\sigma\rangle=v=F_{\pi},
$$

where $F_{\pi} \approx 93 \mathrm{MeV}$ denotes the pion decay constant. The ground state specified in Eq. (12) breaks the $O(4)$ symmetry down to $O(3)$ since the vacuum is invariant only under the rotations of the pion fields. The quark sector of the QM model is described by the Lagrangian density

$$
\mathcal{L}_{\text {quarks }}=\bar{\psi}\left(i \partial_{\mu} \gamma^{\mu}-G\left(\sigma+i \gamma_{5} \pi \cdot \tau\right)\right) \psi,
$$

where $\tau$ are Pauli matrices in the flavor space. In the ground state (12) quarks get a dynamical (that is, a constituent) mass given by

$$
M_{q}=G\langle\sigma\rangle=G F_{\pi} .
$$

We notice that in Eq. (13) there is no explicit mass term for the quarks. As a matter of fact, in this effective model the explicit breaking of chiral symmetry is achieved by the term

$$
\mathcal{L}_{\text {mass }}=h \sigma
$$

in Eq. (11). In the limit $h / F_{\pi}^{3} \ll 1$ this implies $F_{\pi} M_{\pi}^{2}=h$. Although in Eq. (13) there is no explicit mass term, quarks get a constituent mass because of the spontaneous breaking of the $O(4)$ symmetry in the meson sector: This implies that the quark chiral condensate can be nonzero. Finally,

$$
\mathcal{L}_{\mathrm{QM}}=\mathcal{L}_{\text {quarks }}+\mathcal{L}_{\text {mesons }} .
$$

The mean field effective potential of the QM model at zero temperature is given by

$$
\Omega=U+\Omega_{q},
$$

where

$$
U=\frac{\lambda}{4}\left(\sigma^{2}+\pi^{2}-v^{2}\right)^{2}-h \sigma
$$

is the classical potential of the meson fields as it can be read from Eq. (11) and

$$
\Omega_{q}=-2 N_{c} N_{f} \int \frac{d^{3} p}{(2 \pi)^{3}} E_{p}
$$

is the one-loop quark contribution, with

$$
E_{p}=\sqrt{p^{2}+M^{2}}, \quad M=G \sigma .
$$

We notice that the quark mass depends on the field $\sigma$; thus Eq. (17) is the effective potential for the $\sigma$ field computed at one loop and after regularization it corresponds to the condensation energy, namely the difference between the energy of the state with $\langle\sigma\rangle \neq 0$ and $\langle\sigma\rangle=0$ at $T=0$.

The quark loop in Eq. (19) is divergent in the ultraviolet (UV) but the QM model is renormalizable; therefore we can apply a standard renormalization procedure to remove this divergence. To this end we introduce the function [45-47]

$$
\Omega_{q}(s)=-2 N_{c} N_{f} \nu^{2 s} \int \frac{d^{3} p}{(2 \pi)^{3}}\left(p^{2}+M^{2}\right)^{\frac{1}{2}-s},
$$

where $s$ is a complex number and $\nu$ carries the dimension of a mass in order to balance the wrong mass dimension of the integrand when $s \neq 0$. The strategy is to compute the above integral for a finite value of $s$, then make analytical continuation to $s=0$. The integral can be performed analytically with the result

$$
\Omega_{q}(s)=-\frac{N_{c} N_{f}}{4 \pi^{3 / 2}} \nu^{2 s} M^{4-2 s} \frac{\Gamma(s-2)}{\Gamma\left(s-\frac{1}{2}\right)},
$$

where $\Gamma$ denotes the standard Euler function. In the limit $s \rightarrow 0^{+}$we get 


$$
\begin{aligned}
\Omega_{q}(s)= & -\frac{N_{c} N_{f}}{2 \pi^{2}}\left[-\frac{M^{4}}{8 s}+\frac{M^{4}}{16}\left(-3+2 \gamma_{E}\right.\right. \\
& \left.\left.+2 \psi(-1 / 2)+4 \log \frac{M}{\nu}\right)\right],
\end{aligned}
$$

where $\psi(z)=d \log \Gamma(z) / d z$ and $\gamma_{E} \approx 0.577$ is the Euler constant. We notice that the UV divergence is now manifest in the analytical structure of $\Omega_{q}(s)$ in the complex plane; namely it appears as a simple pole at $s=0$.

We now add two counterterms,

$$
\Omega_{\mathrm{ct}}=\frac{\delta v}{2} \sigma^{2}+\frac{\delta \lambda}{4} \sigma^{4},
$$

to the quark loop and require that the renormalized quark loop does not shift the values of the $\sigma$ meson mass as well as the value of the condensate obtained within the classical potential (12); $\delta v$ and $\delta \lambda$ will absorb the divergence of $\Omega_{q}$ and the final result will be a convergent quantity. By defining the renormalized potential as

$$
\Omega_{q}^{\mathrm{ren}}=\Omega_{q}+\Omega_{\mathrm{ct}}
$$

the renormalization conditions thus read

$$
\left.\frac{\partial \Omega_{q}^{\mathrm{ren}}}{\partial \sigma}\right|_{\sigma=v}=0,\left.\quad \frac{\partial^{2} \Omega_{q}^{\mathrm{ren}}}{\partial \sigma^{2}}\right|_{\sigma=v}=0 ;
$$

as a matter of fact, the first condition implies that $\Omega_{q}^{\text {ren }}$ does not shift the global minimum of the effective potential, and this will be given by the minimum of the classical potential, while the second condition implies that for $\sigma=v$, namely in the ground state, the mass matrix of the $\sigma$ meson is not affected by the quark loop. The counterterms can be computed very easily and their expression is not necessary here; therefore we write directly the expression for the renormalized potential, namely

$\Omega_{q}^{\mathrm{ren}}=\frac{3 G^{4}}{32 \pi^{2}} N_{c} N_{f} \sigma^{4}-\frac{G^{4} F_{\pi}^{2}}{8 \pi^{2}} N_{c} N_{f} \sigma^{2}+\frac{G^{4} N_{c} N_{f}}{8 \pi^{2}} \sigma^{4} \log \frac{F_{\pi}}{\sigma}$.

We notice that $\Omega_{q}^{\text {ren }}=0$ for $\sigma=0$ and that $\Omega_{q}^{\text {ren }}=$ $-G^{4} F_{\pi}^{4} N_{c} N_{f} / 32 \pi^{2}$ for $\sigma=F_{\pi}$ : Thus, $\Omega_{q}^{\text {ren }}$ lowers the energy difference between the states with and without a condensate.

The finite temperature thermodynamic potential is finite and does not need any particular treatment: It is given by the standard relativistic fermion gas contribution, namely

$\Omega_{T}=-2 N_{c} N_{f} T \int \frac{d^{3} p}{(2 \pi)^{3}} \log \left(1+e^{-\beta\left(E_{p}-\mu\right)}\right)\left(1+e^{-\beta\left(E_{p}+\mu\right)}\right)$,

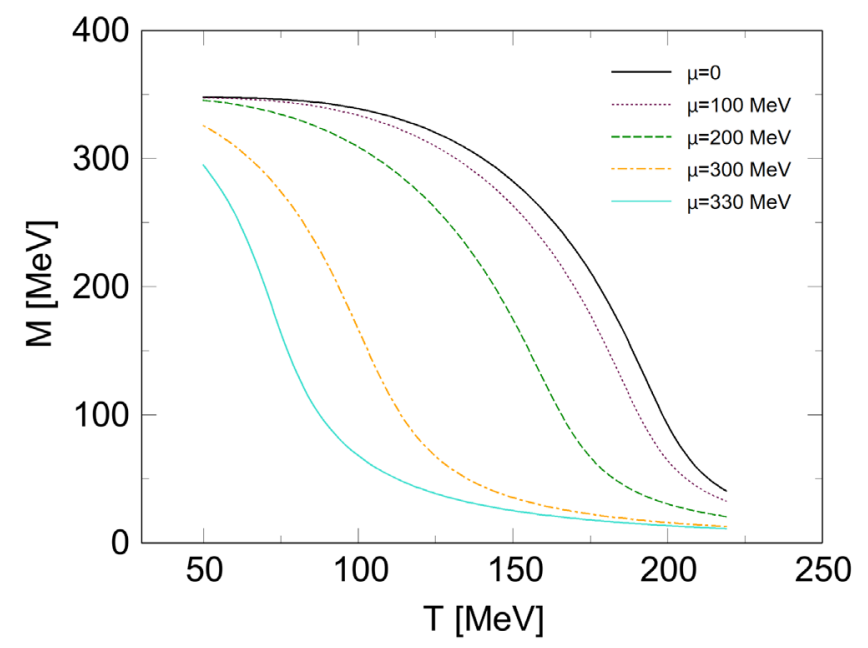

FIG. 2. Constituent quark mass, $M$, versus temperature for several values of the quark chemical potential.

where $\mu$ corresponds to the chemical potential and $E_{p}=$ $\sqrt{p^{2}+M^{2}}$ with $M=g \sigma$. Putting it all together we get

$$
\begin{aligned}
\Omega= & U(\sigma)+\frac{3 G^{4}}{32 \pi^{2}} N_{c} N_{f} \sigma^{4}-\frac{G^{4} F_{\pi}^{2}}{8 \pi^{2}} N_{c} N_{f} \sigma^{2} \\
& +\frac{G^{4} N_{c} N_{f}}{8 \pi^{2}} \sigma^{4} \log \frac{F_{\pi}}{\sigma} \\
& -2 N_{c} N_{f} T \int \frac{d^{3} p}{(2 \pi)^{3}} \log \left(1+e^{-\beta\left(E_{p}-\mu\right)}\right)\left(1+e^{-\beta\left(E_{p}+\mu\right)}\right) .
\end{aligned}
$$

The pressure is $P(T, \mu)=-\Omega(T, \mu, \bar{\sigma})$ where $\bar{\sigma}$ is the value of $\sigma$ that minimizes $\Omega$ at a given $(T, \mu)$.

\section{RESULTS}

\section{A. The thermodynamic curvature}

In this section we summarize the results we have obtained for the QM model. Our parameter set is $M_{\sigma}=$ $700 \mathrm{MeV}, v=F_{\pi}=93 \mathrm{MeV}, M_{\pi}=138 \mathrm{MeV}$ and $G=3.6$; these give $M=335 \mathrm{MeV}$ at $T=0$ and $\mu=0, \lambda=$ $M_{\sigma}^{2} / 2 F_{\pi}^{2}=28.3$ and $h=M_{\pi}^{2} F_{\pi}=1.78 \times 10^{6} \mathrm{MeV}^{3}$. We have also used another parameter set with $M_{\sigma}=600 \mathrm{MeV}$ and $M=350 \mathrm{MeV}$ at $\mu=T=0$ but the results are unchanged qualitatively; therefore we present here only the results related to the first parameter set.

In Fig. 2 we plot the constituent quark mass as a function of the temperature for several values of the quark chemical potential: black solid line is for $\mu=0$, brown dotted line denotes $\mu=100 \mathrm{MeV}$, green dashed line stands for $\mu=200 \mathrm{MeV}$, orange dot-dashed line corresponds to $\mu=300 \mathrm{MeV}$ and finally the turquoise solid line stands for $\mu=330 \mathrm{MeV}$. For any value of $\mu$ there exists a range of temperature in which $M$ decreases: This is the chiral crossover from a low temperature phase with spontaneous 


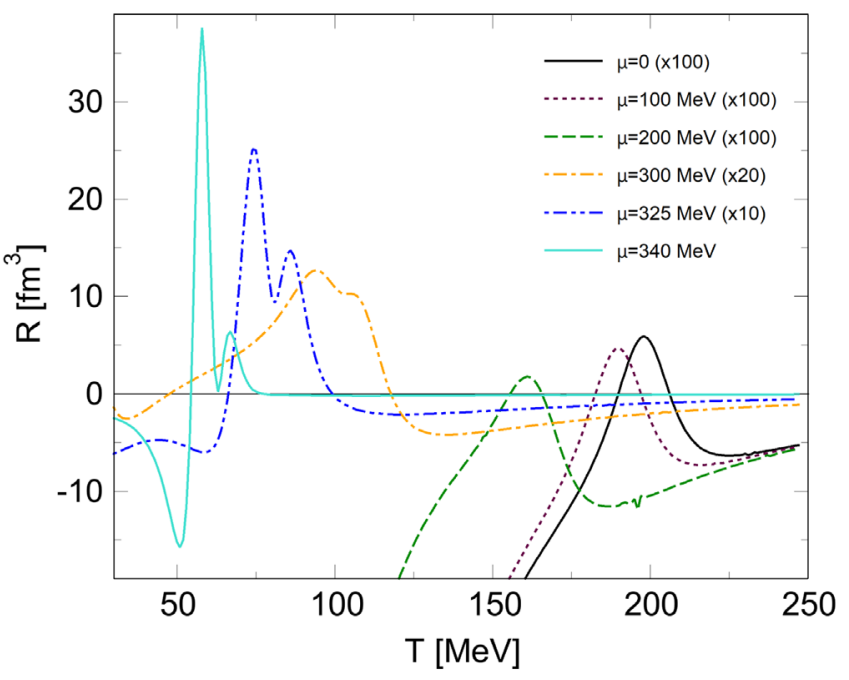

FIG. 3. Thermodynamic curvature, $R$, versus temperature for several values of the quark chemical potential.

chiral symmetry breaking from a high temperature phase in which chiral symmetry is approximately restored. The larger $\mu$ the sharper the change of $M$ with $T$ is, and for $(T, \mu) \equiv\left(T_{E}, \mu_{E}\right) \approx(30,360 \mathrm{MeV})$ we find the CEP at which the crossover becomes a true second order phase transition and for $\mu>\mu_{E}$ the phase transition is a first order one.

In Fig. 3 we plot the thermodynamic curvature, $R$, versus the temperature for several values of the quark chemical potential: black solid line is for $\mu=0$, brown dotted line denotes $\mu=100 \mathrm{MeV}$, green dashed line stands for $\mu=200 \mathrm{MeV}$, orange dot-dashed line corresponds to $\mu=300 \mathrm{MeV}$, blue dot-dot-dashed line denotes $\mu=$ $325 \mathrm{MeV}$ and finally the turquoise solid line stands for $\mu=340 \mathrm{MeV}$. At small temperature the curvature is negative, as for a free fermion gas. However, we notice that the sign of $R$ changes around the crossover, then becoming negative again for $T \gg T_{c}$ : The crossover corresponds to a change in the geometry from hyperbolic to elliptic [60-63]. Following [43] we identify the region in which $R>0$ with the crossover. This interpretation is supported by the fact that the local maxima of $R$ appear to be very close to those of $|d M / d T|$, the latter giving a rough location of the crossover itself; see also below. The fact that the magnitude of $R$ in the critical region remains small for small $\mu$ is related to the fact that in this region the crossover is very smooth; on the other hand, when we approach the critical end point the crossover is closer to a second order phase transition and $R$ develops clear peaks.

We also notice that the structure of $R(T)$ as the critical end point is approached is quite interesting. Indeed, for $\mu=340 \mathrm{MeV}$ in Fig. 3 we find that $R$ is negative and drops down before rising to a positive peak around the crossover. The behavior of $R$ that we find can be understood mathematically since $R$ combines several second and third order cumulants with different signs; see Sec. II. Overall, the increase of the magnitude of $R$ as the CEP is approached is due to the determinant of the metric that becomes small around the CEP and eventually vanishes at the CEP; see below. We also notice that increasing the temperature right above the peak results in $R=0$ then $R$ stays positive for a substantial temperature range, before becoming negative again: The $R=0$ point can be understood since $\phi_{, \beta \beta \beta}, \phi_{, \beta \beta \gamma}$, $\phi_{, \beta \gamma \gamma}$ and $\phi_{, \gamma \gamma \gamma}$ vanish at that temperature and so $R$ does. This is in agreement with the well-known fact that the third order cumulants change sign around the critical end point [64-67]

\section{B. The thermodynamic geometry at the critical line}

In the model at hand, as well as in full QCD, there is no real phase transition at high temperature and small chemical potential, rather only a smooth crossover. Because of this, the location of the transition temperature is ambiguous: For example, the crossover region can be identified around the temperature at which $|d M / d T|$ is maximum, or by the location of the peak of the chiral susceptibility. We define two critical temperatures:

$$
\begin{gathered}
T_{c 1} \text { : from the peak of }\left|\frac{d M}{d T}\right|, \\
T_{c 2} \text { : from the positive peak of } R .
\end{gathered}
$$

In particular, using $T_{c 1}$ we define the crossover at a given $\mu$ by choosing the temperature at which the constituent quark mass has its maximum change. In Fig. 4 we plot $T_{c 1}$ and $T_{c 2}$ as a function of the chemical potential. The two lines end up and coincide at the critical end point, which is denoted by an indigo dot. The two transition temperatures differ a few percent at most; therefore the local maxima of $R$ in the $(T, \mu)$ plane are very close to the points at which the constituent quark mass has its maximum change which

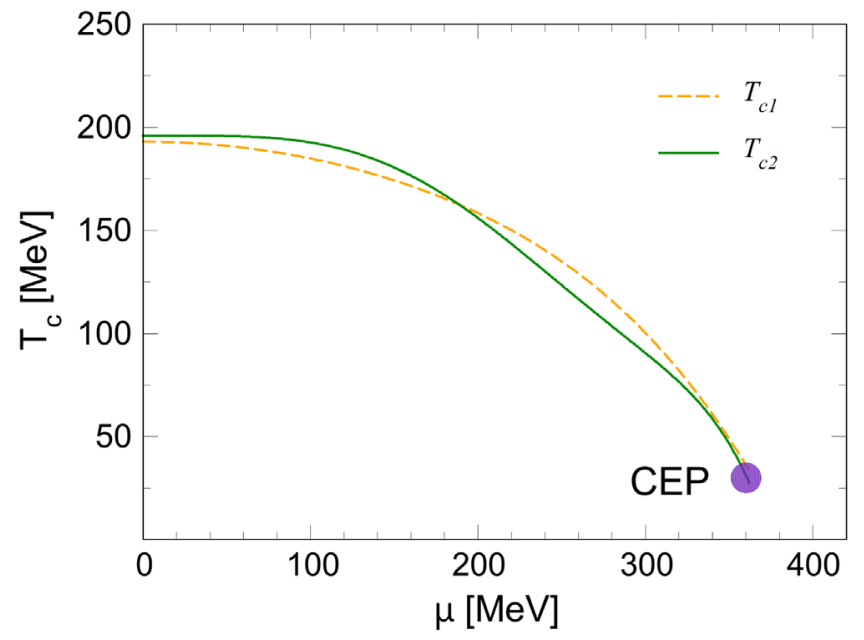

FIG. 4. Transition temperatures, $T_{c 1}$ and $T_{c 2}$, as a function of the chemical potential. The indigo dot denotes the CEP. 

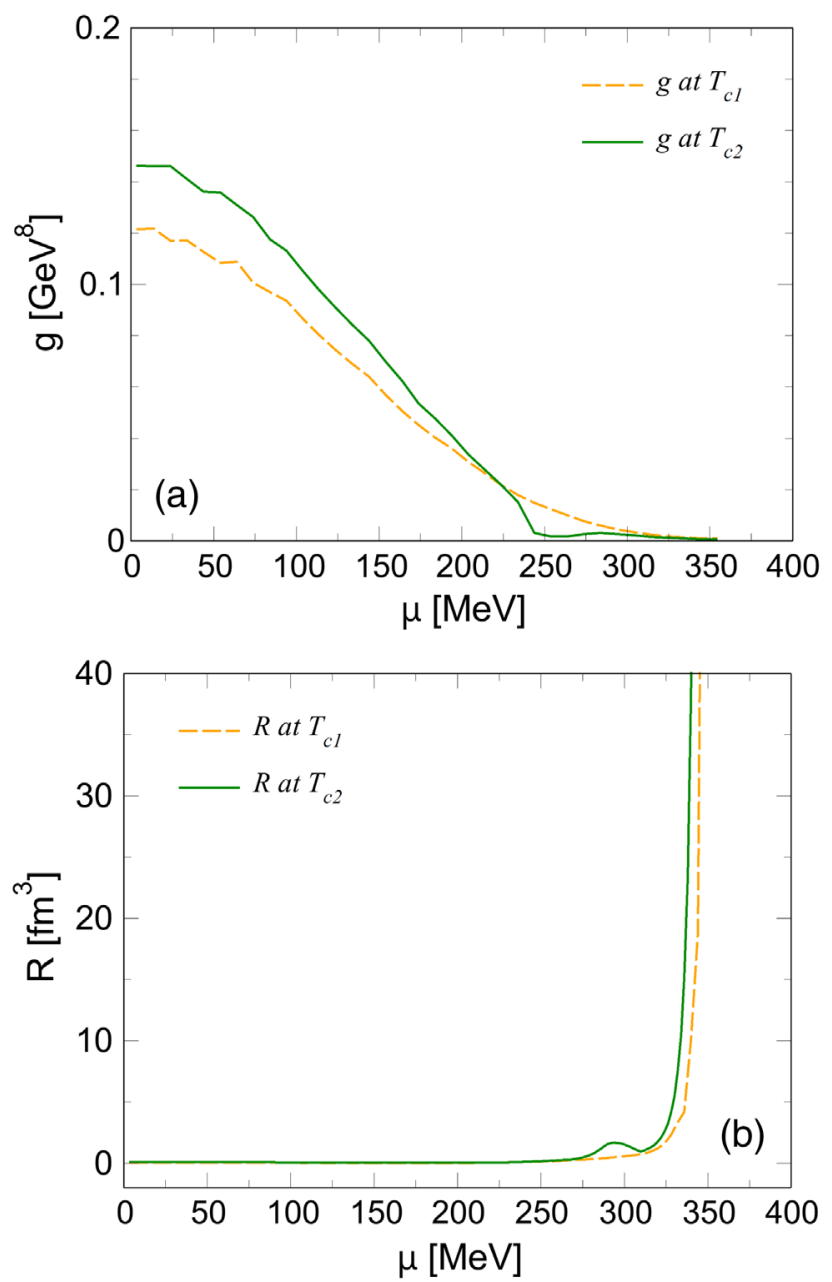

FIG. 5. (a) Determinant of the metric as a function of the chemical potential in two cases. (b) Thermodynamic curvature versus temperature.

supports the idea that the peaks of $R$ do relate to the chiral crossover.

In Fig. 5(a) we plot the determinant of the metric, $g$, in the proximity of the critical line. The orange dashed line corresponds to the value of $g$ computed at $T_{c 1}$, while the solid green line denotes the values of $g$ computed at $T_{c 2}$. We notice that $g$ is always positive in the crossover region; hence the thermodynamic distance is well defined there and the system is thermodynamically stable. The mismatch between the two curves is clearly related to the definition used for the critical temperature; nevertheless, the qualitative behavior of $g$ is the same in the two cases. We also find that around the CEP the determinant is very small and eventually vanishes at the CEP, as anticipated: The vanishing of the determinant at the CEP is expected at a second order phase transition on the base of thermodynamic stability [68]. Moreover, because $g=0$ at the CEP we get that $R$ diverges there, as it happens for example for the van der Waals gas $[37,41,69]$.
In Fig. 5(b) we plot $R$ at the critical temperature. Again, we compare the result obtained using two different definitions of the critical temperature: The orange dashed line denotes $R$ computed at $T_{c 1}$, while the solid green line denotes the values of $R$ computed at $T_{c 2}$. We notice that in both cases the qualitative behavior of $R$ is the same. In particular, the magnitude of $R$ increases when $(T, \mu)$ approach the CEP and diverges at the CEP, in agreement with the previous discussion. The divergence of $R$ at the CEP supports the idea that $|R|$ measures the correlation volume around the phase transition since the latter also diverges at the CEP [3].

The thermodynamic curvature diverges at the CEP because the determinant of the metric is zero there: The condition $g=0$ corresponds to thermodynamic instability and thus to a phase transition. Clearly we can write (see Sec. II for more details)

$$
\begin{aligned}
g & =g_{\beta \beta} g_{\gamma \gamma}-g_{\beta \gamma}^{2} \\
& =\left\langle(\Delta U)^{2}\right\rangle\left\langle(\Delta N)^{2}\right\rangle-\langle\Delta U \Delta N\rangle^{2},
\end{aligned}
$$

where in particular $\langle\Delta U \Delta N\rangle$ corresponds to the mixed energy-baryon number fluctuation. In Fig. 6 we plot $T_{c 2}^{4}\left(g_{\beta \beta} g_{\gamma \gamma}\right)^{1 / 2}$ (indigo dot-dashed line) and $T_{c 2}^{4} g_{\beta \gamma}$ (orange solid line) as a function of the chemical potential along $T_{c 2}$ (for $T_{c 1}$ we get similar results therefore we do not show them here). At $\mu=0$ we find $g_{\beta \gamma}=0$ thus $g>0$; as $\mu$ is increased the mixed susceptibility rapidly grows up and eventually hits $\left(g_{\beta \beta} g_{\gamma \gamma}\right)^{1 / 2}$ leading to $g=0$ and to the divergent curvature. We conclude that the CEP (i.e., the divergent curvature) occurs in the phase diagram because the underlying microscopic interaction leads to a rapidly increasing mixed energy and baryon number fluctuation. We notice that the vanishing of $g$ is something more than

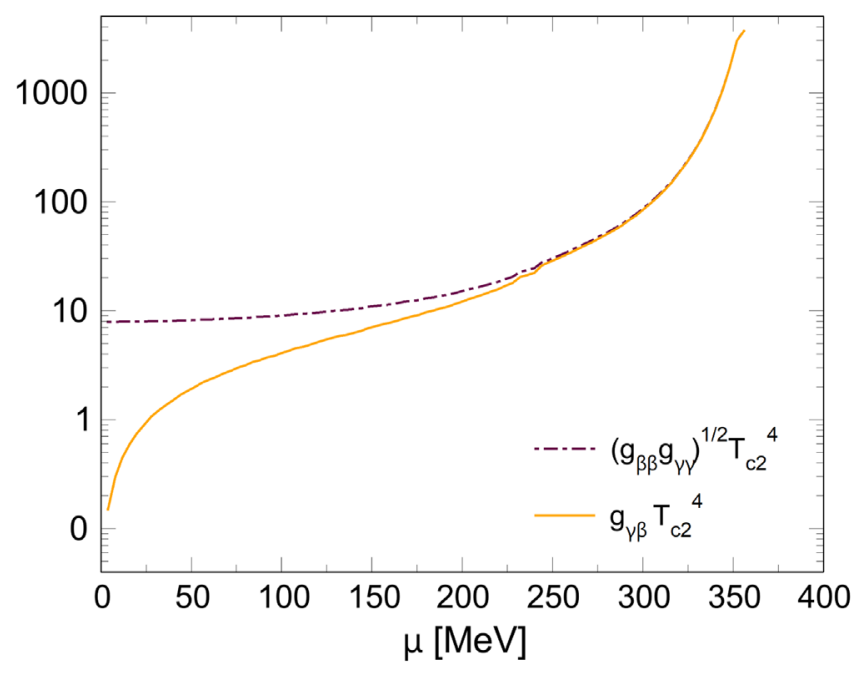

FIG. 6. $\quad T_{c 2}^{4}\left(g_{\beta \beta} g_{\gamma \gamma}\right)^{1 / 2}$ and $T_{c 2}^{4} g_{\beta \gamma}$ as a function of the chemical potential along $T_{c 2}$. 

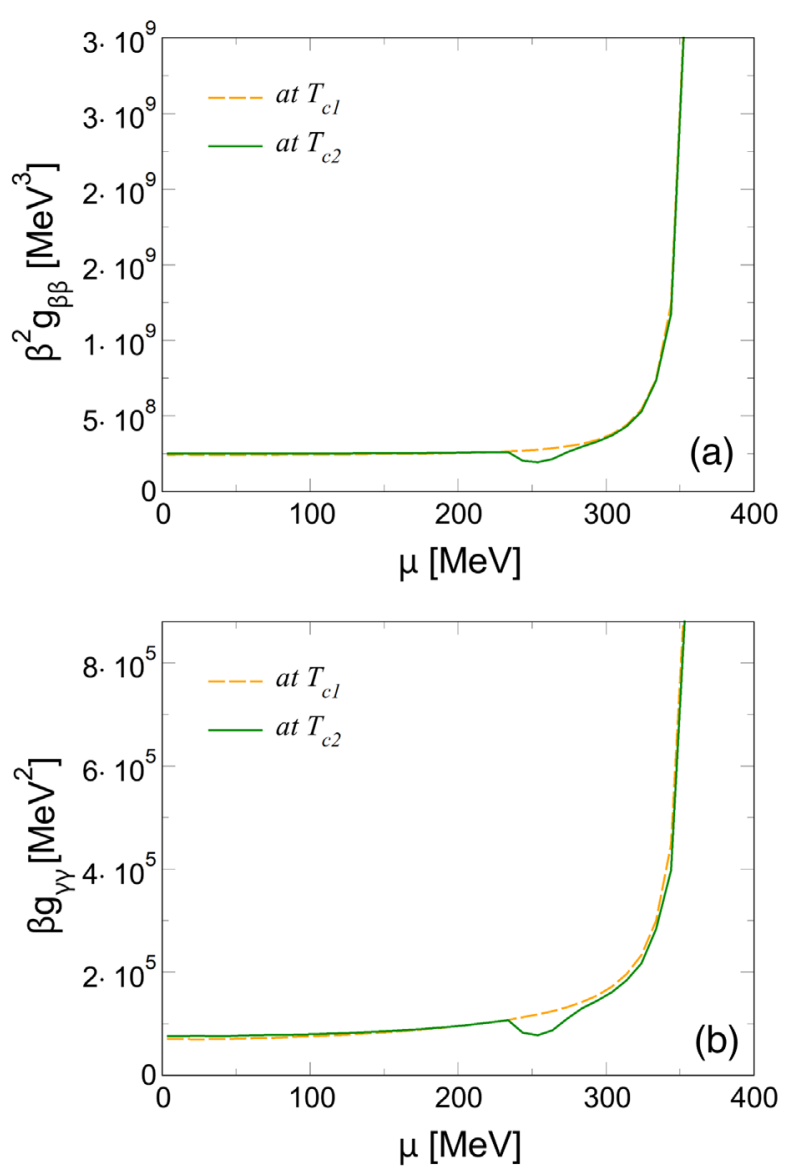

FIG. 7. Specific heat [panel (a)], isothermal compressibility [panel (b)] at the critical line.

getting a divergent baryon number susceptibility at the CEP: In fact, at the CEP all the matrix elements of the metric diverge, but it is the vanishing of the determinant that guarantees that $R$ diverges and thus the crossover becomes a second order phase transition. We will discuss how the mixed susceptibility is sensitive to the location of the CEP in a forthcoming article.

In Fig. 7(a) we plot $\beta^{2} g_{\beta \beta}$ and in Fig. 7(b) we plot $\beta g_{\gamma \gamma}$ at the critical line. The orange dashed line corresponds to data computed at $T_{c 1}$ while the green solid line denotes data computed at $T_{c 2}$. According to Eqs. (9) and (10), $\beta^{2} g_{\beta \beta}$ and $\beta g_{\gamma \gamma}$ are proportional to the specific heat and the isothermal compressibility respectively. We notice that both quantities stay finite around the crossover at small $\mu$ but diverge as the critical end point is approached, in agreement with the fact that crossover becomes a second order phase transition there.

\section{SUMMARY AND CONCLUSIONS}

We have applied the concept of thermodynamic geometry, in particular of thermodynamic curvature $R$, to the chiral crossover of QCD at finite temperature $T$ and finite baryon chemical potential $\mu$. The crossover has been modeled by the renormalized QM model which is capable of describing the spontaneous breaking chiral symmetry. Although thermodynamic geometry was introduced many years ago, its use for the high temperature phase of QCD has been only marginal. One of the merits of $R$ is that $|R| \propto \xi^{3}$ near a second order phase transition in three spatial dimensions, where $\xi$ corresponds to the correlation length. In QCD a crossover is expected at high temperature instead of a real second order phase transition; therefore the interpretation of $R$ has to be done carefully. Nevertheless it is fair to relate the peaks of $R$ to the chiral crossover. We support this idea here, albeit there are some details that in our opinion deserve further study.

We have studied $R$ for the QM model at finite $T$ and $\mu$. In the QM model the mass constituent quark mass, $M=M(T, \mu)$, is related to the quark condensate and it is computed self-consistently. We have found that for small values of $\mu$, where the model presents a very smooth crossover at finite temperature, increasing the temperature results in a change of sign from negative to positive in the crossover region, as well as to a modest peak of $R$. This is similar to what has been observed in the Nambu-JonaLasinio model [43], and this peak appears in correspondence to the peak of $|d M / d T|$ so it is natural to identify the peak of $R$ with the chiral crossover. The change of the sign of $R$ has been interpreted previously as an indicator of the attractive/repulsive nature of the mesoscopic interaction. This suggests that around the crossover, the interaction becomes attractive, while the fermion nature of the system dominates at low and high temperature and the curvature is negative. We have also studied several matrix elements of the metric, which are related to the isothermal compressibility and to the specific heat, as well as the curvature, at the critical line $T_{c}\left(\mu_{c}\right)$, finding the divergence of these as the critical end point is approached. Overall, these results support the idea that although in QCD at small $\mu$ there is a smooth crossover rather than a phase transition, the thermodynamic curvature is capable of capturing this crossover by developing local maxima around $T_{c}$.

We have also pointed out that due to fluctuations of both energy and baryon number in the grand-canonical ensemble, a mixed susceptibility, $\langle\Delta U \Delta N\rangle$, develops at finite $\mu$. We have shown that the CEP in the temperature and baryon chemical potential plane occurs when the determinant of the thermodynamic metric vanishes: This happens as $\langle\Delta U \Delta N\rangle$ grows up considerably at the finite baryon chemical potential.

There are several aspects that deserve further investigation. First, it is interesting to study how the repulsive vector interaction affects $R$ at finite $T$ and $\mu$ : This indeed might shed some light on the connection between the attractive/ repulsive nature of the interaction and the sign of $R$. To this end, it is useful to remark that effective models of QCD like the one used in this article are ideal tools to study the thermodynamic curvature, for at least two reasons. The first 
one is that the interaction is strong, so they allow us to study quantitatively $R$ for systems that are quite far from the ideal gas or the weakly coupled system: In fact, most of the works done on $R$ in the last $\approx 40$ years hardly consider strongly coupled systems; therefore a fundamental understanding of $R$ for these thermodynamic systems is lacking. In addition to this, the microscopic interaction in these models is under control, so it is possible to study how the microscopic details affect $R$ around the phase transition. Moreover, it is of certain interest to study $R(T, \mu)$ around the QCD chiral crossover using a Ginzburg-Landau effective potential, since this might lead to analytical expressions of the curvature and help to prove quantitatively the relation $|R| \propto \xi^{3}$ for the model at hand. Even more, it is certainly interesting to study the behavior of $R$ for higherdimensional varieties, for example enlarging the present two-dimensional space by a third direction representing isospin or magnetic field. Finally, it is well known that the QCD phase structure at large density is pretty rich: It is interesting to apply the ideas of thermodynamic curvature in this regime as well. All these interesting themes might not shed new light on the phase structure of QCD, but we are confident that they will help us to understand more about the significance of the thermodynamic geometry.

Moreover, the thermodynamic geometry allows for a natural definition of the CEP since this can be identified with the point in the $(T, \mu)$ plane where the determinant of the metric vanishes, which is equivalent to a precise relation between susceptibilities at the CEP. It will be interesting to study how the susceptibilities at small and moderate $\mu$ are sensitive to the location of the CEP, hopefully to get information that can be tested in first principle calculations and shed light on the CEP in full QCD. We plan to report on this in a forthcoming article.

\section{ACKNOWLEDGMENTS}

The authors acknowledge Paolo Castorina, Daniele Lanteri, John Petrucci and Sijiang Yang for inspiration, discussions and comments on the first version of this article. The work of M. R. is supported by the National Science Foundation of China (Grants No. 11805087 and No. 11875153) and by the Fundamental Research Funds for the Central Universities (Grant No. 862946).
[1] F. Weinhold, J. Chem. Phys. 63, 2479 (1975).

[2] F. Weinhold, J. Chem. Phys. 63, 2484 (1975).

[3] G. Ruppeiner, Phys. Rev. A 20, 1608 (1979).

[4] G. Ruppeiner, Phys. Rev. A 24, 488 (1981).

[5] G. Ruppeiner, Phys. Rev. A 27, 1116 (1983).

[6] G. Ruppeiner, Phys. Rev. Lett. 50, 287 (1983).

[7] G. Ruppeiner, Phys. Rev. A 31, 2688 (1985).

[8] G. Ruppeiner, Phys. Rev. A 32, 3141 (1985).

[9] G. Ruppeiner, Phys. Rev. A 34, 4316 (1986).

[10] G. Ruppeiner and D. Christopher, Phys. Rev. A 41, 2200 (1990).

[11] G. Ruppeiner and J. Chance, J. Chem. Phys. 92, 3700 (1990).

[12] G. Ruppeiner, Phys. Rev. A 44, 3583 (1991).

[13] G. Ruppeiner, Phys. Rev. E 47, 934 (1993).

[14] G. Ruppeiner, Advances in Thermodynamics, edited by S. Sieniutycz, P. Salamon, and G. A. Mansoori (Taylor and Francis, New York, 1990), Vol. 3, p. 129.

[15] G. Ruppeiner, Rev. Mod. Phys. 67, 605 (1995).

[16] G. Ruppeiner, Phys. Rev. E 57, 5135 (1998).

[17] G. Ruppeiner, Phys. Rev. E 72, 016120 (2005).

[18] G. Ruppeiner, Phys. Rev. D 78, 024016 (2008).

[19] G. Ruppeiner, Am. J. Phys. 78, 1170 (2010).

[20] G. Ruppeiner, Phys. Rev. E 86, 021130 (2012).

[21] G. Ruppeiner, A. Sahay, T. Sarkar, and G. Sengupta, Phys. Rev. E 86, 052103 (2012).

[22] G. Ruppeiner, J. Phys. Conf. Ser. 410, 012138 (2013).

[23] H. May, P. Mausbach, and G. Ruppeiner, Phys. Rev. E 88, 032123 (2013).

[24] G. Ruppeiner, J. Low Temp. Phys. 174, 13 (2014).
[25] G. Ruppeiner and S. Bellucci, Phys. Rev. E 91, 012116 (2015).

[26] S. Bellucci and B. N. Tiwari, Physica (Amsterdam) 390A, 2074 (2011).

[27] H. May, P. Mausbach, and G. Ruppeiner, Phys. Rev. E 91, 032141 (2015).

[28] G. Ruppeiner, P. Mausbach, and H. May, Phys. Lett. A 379, 646 (2015).

[29] G. Ruppeiner, J. Low Temp. Phys. 185, 246 (2016).

[30] G. Ruppeiner, N. Dyjack, A. McAloon, and J. Stoops, J. Chem. Phys. 146, 224501 (2017).

[31] S. Wei and Y. Liu, Phys. Rev. D 87, 044014 (2013).

[32] B. Mirza and H. Mohammadzadeh, Phys. Rev. E 78, 021127 (2008).

[33] B. Mirza and H. Mohammadzadeh, Phys. Rev. E 80, 011132 (2009).

[34] P. Castorina, M. Imbrosciano, and D. Lanteri, Phys. Rev. D 98, 096006 (2018).

[35] A. Sahay and R. Jha, Phys. Rev. D 96, 126017 (2017).

[36] H. Janyszek and R. Mrugala, J. Phys. A 23, 467 (1990).

[37] H. Janyszek, J. Phys. A 23, 477 (1990).

[38] H. Janyszek and R. Mrugala, Phys. Rev. A 39, 6515 (1989).

[39] H. Oshima, T. Obata, and H. Hara, J. Phys. A 32, 6373 (1999).

[40] L. Diosi and B. Lukacs, Phys. Rev. A 31, 3415 (1995).

[41] D. Brody and N. Rivier, Phys. Rev. E 51, 1006 (1995).

[42] P. Castorina, M. Imbrosciano, and D. Lanteri, Eur. Phys. J. Plus 134, 164 (2019).

[43] P. Castorina, D. Lanteri, and S. Mancani, arXiv:1905.05296.

[44] M. E. Crooks, Phys. Rev. Lett. 99, 100602 (2007). 
[45] M. Ruggieri, M. Tachibana, and V. Greco, J. High Energy Phys. 07 (2013) 165.

[46] M. Ruggieri, L. Oliva, P. Castorina, R. Gatto, and V. Greco, Phys. Lett. B 734, 255 (2014).

[47] M. Frasca and M. Ruggieri, Phys. Rev. D 83, 094024 (2011).

[48] V. Skokov, B. Friman, E. Nakano, K. Redlich, and B.-J. Schaefer, Phys. Rev. D 82, 034029 (2010).

[49] S. Borsanyi, Z. Fodor, C. Hoelbling, S. D. Katz, S. Krieg, C. Ratti, and K. K. Szabó (Wuppertal-Budapest Collaboration), J. High Energy Phys. 09 (2010) 073.

[50] S. Borsanyi, G. Endrodi, Z. Fodor, A. Jakovac, S. D. Katz, S. Krieg, C. Ratti, and K. K. Szabo, J. High Energy Phys. 11 (2010) 077.

[51] M. Cheng et al., Phys. Rev. D 81, 054504 (2010).

[52] A. Bazavov et al., Phys. Rev. D 85, 054503 (2012).

[53] S. Borsanyi, Z. Fodor, C. Hoelbling, S. D. Katz, S. Krieg, and K. K. Szabo, Phys. Lett. B 730, 99 (2014).

[54] A. Bzdak, S. Esumi, V. Koch, J. Liao, M. Stephanov, and N. $\mathrm{Xu}$, arXiv:1906.00936.

[55] K. Fukushima and T. Hatsuda, Rep. Prog. Phys. 74, 014001 (2011).

[56] R. K. Pathria and P. B. Beale, Statistical Mechanics, 3rd ed. (Academic Press, New York, 2011).

[57] L. D. Landau and E. M. Lifshitz, Course of Theoretical Physics (Butterworth-Heinemann, Washington, DC, 1980), Vol. 5.
[58] M. E. Peskin and D. V. Schroeder, An Introduction to Quantum Field Theory (Westview Press, Boulder Colorado, 1995).

[59] S. Weinberg, The Quantum Theory of Fields, Modern Applications Vol. 2 (Cambridge University Press, Cambridge, England, 1996).

[60] G. G. Saccheri, Euclides ab Omni Naevo Vindicatus (1733) [Girolamo Saccheri's Euclides Vindicatus, edited by G. B. Halsted (Open Court Publishing Company, Chicago, 1920)].

[61] E. Beltrami, Gior. Mat. 6, 248 (1868).

[62] N. Lobachevski, Geometrical Researches on the Theory of Parallels, translated by George Bruce Halsted (University of Michigan Library, Ann Arbor, MI, 1892).

[63] B. A. Dubrovin, A. T. Fomenko, and S. P. Novikov, Modern Geometry: Methods and Applications (Springer, New York, 1984).

[64] V. Vovchenko, D. V. Anchishkin, M. I. Gorenstein, and R. V. Poberezhnyuk, Phys. Rev. C 92, 054901 (2015).

[65] Z. Li, Y. Chen, D. Li, and M. Huang, Chin. Phys. C 42, 013103 (2018).

[66] Z. Li, K. Xu, X. Wang, and M. Huang, Eur. Phys. J. C 79, 245 (2019).

[67] G. y. Shao, Z. d. Tang, X. y. Gao, and W. b. He, Eur. Phys. J. C 78, 138 (2018).

[68] H. B. Callen, Thermodynamics and Introduction to Thermostatistics, 2nd ed. (John Wiley \& Sons, New York, 1985).

[69] M. Santoro and S. Preston, arXiv:math-ph/0505010. 